\title{
Machine Learning Techniques for Quantification of Knee Segmentation from MRI
}

\author{
Sujeet More $\mathbb{D}^{\mathbb{D}},{ }^{1}$ Jimmy Singla $\mathbb{D}^{1},{ }^{1}$ Ahed Abugabah, ${ }^{2}$ and Ahmad Ali AlZubi ${ }^{3}$ \\ ${ }^{1}$ School of Computer Science and Engineering, Lovely Professional University, Kapurthala, India \\ ${ }^{2}$ College of Technological Innovation, Zayed University, Dubai, UAE \\ ${ }^{3}$ Computer Science Department, King Saud University, Riyadh, Saudi Arabia \\ Correspondence should be addressed to Jimmy Singla; jimmy.21733@lpu.co.in
}

Received 26 October 2020; Revised 16 November 2020; Accepted 27 November 2020; Published 7 December 2020

Academic Editor: Dr. Shahzad Sarfraz

Copyright ( $\odot 2020$ Sujeet More et al. This is an open access article distributed under the Creative Commons Attribution License, which permits unrestricted use, distribution, and reproduction in any medium, provided the original work is properly cited.

\begin{abstract}
Magnetic resonance imaging (MRI) is precise and efficient for interpreting the soft and hard tissues. Moreover, for the detailed diagnosis of varied diseases such as knee rheumatoid arthritis (RA), segmentation of the knee magnetic resonance image is a challenging and complex task that has been explored broadly. However, the accuracy and reproducibility of segmentation approaches may require prior extraction of tissues from MR images. The advances in computational methods for segmentation are reliant on several parameters such as the complexity of the tissue, quality, and acquisition process involved. This review paper focuses and briefly describes the challenges faced by segmentation techniques from magnetic resonance images followed by an overview of diverse categories of segmentation approaches. The review paper also focuses on automatic approaches and semiautomatic approaches which are extensively used with performance metrics and sufficient achievement for clinical trial assistance. Furthermore, the results of different approaches related to MR sequences used to image the knee tissues and future aspects of the segmentation are discussed.
\end{abstract}

\section{Introduction}

Arthritis is one of the serious, prevalent joint diseases that cause disability and health issues in a large population. This arthritis is categorized with progressive degradation of joint tissues with a variety of abnormalities [1] and is a serious issue in recent years. Nearly sixty to seventy percent of people older than 60 years suffer from arthritis $[2,3]$. Osteoarthritis is the damage of joint cartilage and leads to damage of functionality in the knee and hips, and the early signs can be observed with tears in cartilage. But rheumatoid arthritis is an autoimmune disease that largely affects the soft tissues around joints, bones, and cartilage. Therefore, the thickness and volume of the knee joint are the important parameters to evaluate rheumatoid arthritis.

Different imaging modalities can be deployed to estimate the quantitative measures of knee arthritis. But the ability of magnetic resonance imaging to provide the quality imaging of bones with soft tissues, cartilage, and tendons as illustrated in Figure 1, for the diagnosis and treatment of diverse diseases, is extensively utilized. Magnetic resonance images provide information about damage and inflammation with more sensitivity than other modalities. The magnetic resonance images may contain hundreds of slices depending on the sample rate. The magnetic resonance images are a valuable instrument for the treatment and study of different ailments. The significant cost of labor, hours for analyzing a single scan by the radiologist, and insensitive for detection of progression of arthritis make the treatment more difficult, expensive, and inefficient.

Regardless of which disease is under study, the processing architecture includes a step known as segmentation for extracting the quantitative measures. The segmentation is a process of selecting the area of interest (AOI) concerning certain characteristics. Let $X, Y$, and $Z$ be a finite grid of $p, q$ pixels with $Y$ set of intensities and $Z$ objects, respectively. 


$$
\begin{aligned}
& X=(x=(p, q): p=0,1, \ldots, p-1, q=0,1, \ldots, q-1) \\
& Y=(0,1, \ldots, y-1) \\
& Z=(0,1, \ldots, z-1)
\end{aligned}
$$

Let $g: X \longrightarrow Y$ and $m: X \longrightarrow Z$ denote the image and an area, respectively. The image segmentation divides an image $g$ into $Z$-connected subimages.

This process of segmentation is important in providing the information about knee structure and the progress of the disease to the radiologist for diagnosis. However, this is a perilous and complex task for numerous reasons such as irregular shape, size, and connecting tissues. Therefore, many studies have focused on the progress of different methods to segment the knee magnetic resonance images $[4,5]$. Although many studies on automatic and semiautomatic approaches are conducted, this segmentation from the magnetic resonance image is a future research problem [6] for the development of fully automated accurate and precise techniques. Thus, this scientific review provides a comprehensive knowledge of different computational methods utilized for the segmentation of magnetic resonance images. The review also focuses on exploring the challenges during segmentation.

Consequently, articles were screened for selection based on title and abstract. Only original papers that are published in journals and conferences are selected. Books, book chapters, reports, and thesis are excluded from selection criteria. Articles that are written in English and focused on rheumatoid arthritis disease are selected for research. Articles written in other than English and not related to other diseases are excluded. Articles using the machine learning approach are included. Articles that entirely use image processing methods are excluded from research.

This scientific article is organized as follows: Section 2 discusses the challenges faced in the segmentation of magnetic resonance images. Section 3 describes the various approaches followed by automated and semiautomated segmentation methods with their metrics in Section 4. Section 5 includes discussion on existing work reviewed in this article. Section 6 accomplishes the conclusion of the review article.

\section{Challenges in Segmentation of Magnetic Resonance Image}

In the recent past, several efforts have been made for the segmentation of magnetic resonance images with the progression of arthritis. Generally, every algorithm is evaluated with different parameters on the image. These algorithms work well with a few sets of data but do not provide efficient performance measures. This is because of different features associated with knee structure that result in difficulties with the segmentation of magnetic resonance images.

2.1. Structure of Tissue. The structure of tissues is measured in terms of thickness. In normal humans, the knee cartilage density is about $2 \mathrm{~mm}$ to $4 \mathrm{~mm}$ with a curled surface with no blood vessels, and this density decreases at the rate of $\sim 2.5 \%$ to $50 \%[7,8]$ every year in rheumatoid arthritis. These structures become thin and irregular in shape and are a challenging task in all slices. However, the edges between tibia and femur cartilage exhibit complications as represented in Figure 2(b), for providing reliable measures.

2.2. Magnetic Field Strength. The signal quantity in the mode of fissile quality is determined in magnetic resonance images. As the frequency of the magnetic field, strength $1.5 \mathrm{~T}$, increases linearly, the signal-to-noise ratio (SNR) is amplified in magnetic resonance imaging [9]. Furthermore, there is proportionality between SNR and scan time. An increase in scan time increases the SNR value and often leads to artifacts in the image. For a high field, i.e., $3 \mathrm{~T}$ or $7 \mathrm{~T}$ strength, the visual quality and segmentation process of cartilage are improved. However, in $3 \mathrm{~T}$ magnetic strength, the T1-weighted images appear to be different than normal, and in $7 \mathrm{~T}$ strength, the T2-weighted images interrupt the scanning process and deploy more artifacts in the image leading to difficulty in finding a region of interest and other features [10].

2.3. Image Artifacts and Properties. MR imaging images are always liable to dissimilar types of artifacts. These relics are represented in the form of the pulse signal, affected volume, and chemical variations that precede an incorrect diagnosis. These artifacts are instigated by the magnetic field, resulting in uneven tissue representation. The chemical variations result in shady and bright spots on the edges of the tissues. These artifacts result in misinterpretation and may wrongly include the area of interest consisting of artifacts. The decreased quality of the edges due to volume effect and variations in signal intensities makes it difficult to develop computational approaches.

The local variation in tissue properties that exhibits several problems within an image is identified as low visibility areas. Figure 3 illustrates the variations and decreasing the edge quality of the knee magnetic resonance image.

As discussed, several issues and challenges need to be considered during the segmentation process for computational efficiency. The above challenges discussed are not only for automatic approaches but also for semiautomatic and manual approaches. The various segmentation techniques are reviewed and examined in the subsequent section.

\section{Knee Tissue Segmentation Approaches}

The knee bone is the major and largest bone within the human body and is the one that is most affected by rheumatoid arthritis disease. The cartilage and bone segments and features are important for the study of this rheumatoid arthritis disease. Recently, bone shape was proposed for predicting the rheumatoid arthritis progression [12]. This bone segmentation is a very critical assessment as connected cartilage degeneration is possible after the reconstruction of ACL [13]. In the class of approaches, the knee cartilage is 


\begin{tabular}{|c|c|}
\hline Inclusion criteria & Exclusion criteria \\
\hline (i) Original articles and conference papers & (i) Books, book chapters, reports, and thesis \\
\hline (ii) Articles published in English & (ii) Articles written in other than English \\
\hline (iii) Disease related to only rheumatoid arthritis & (iii) Disease other than rheumatoid arthritis \\
\hline (iv) Diagnosis using machine learning approach & (iv) Articles using completely image processing approach \\
\hline (v) Articles only focused on diagnosis & (v) Articles related to treatment \\
\hline
\end{tabular}

Figure 1: Inclusion and exclusion criteria for selecting papers.

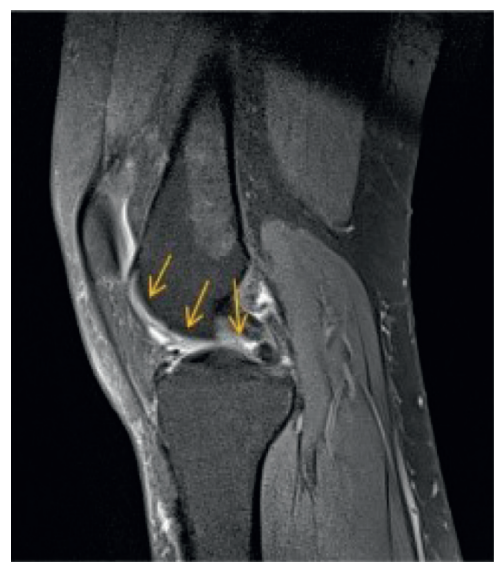

(a)

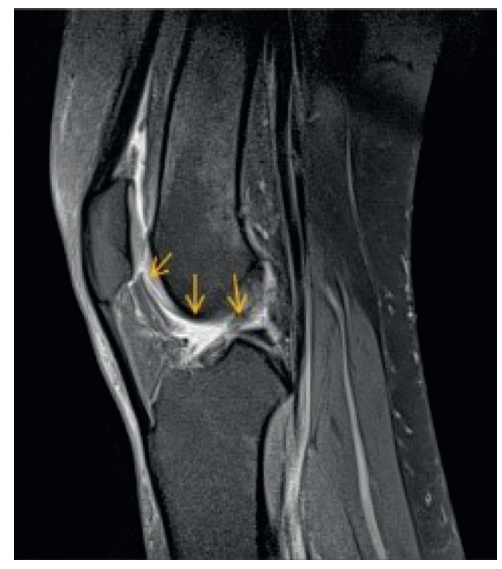

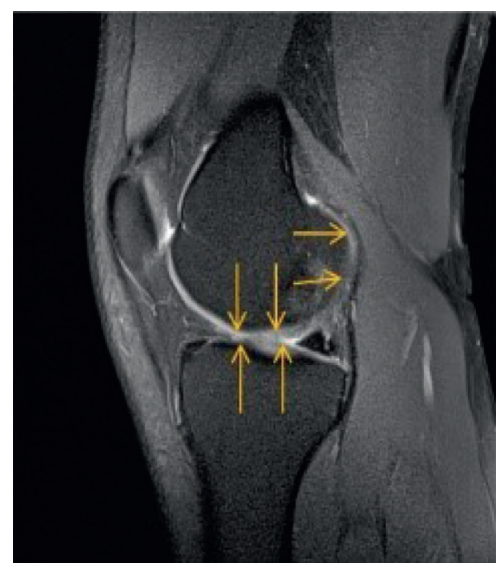

(b)

FIgUre 2: (a) Irregular cartilage tissue. (b) Diffused edges between the tibia and femur bone.

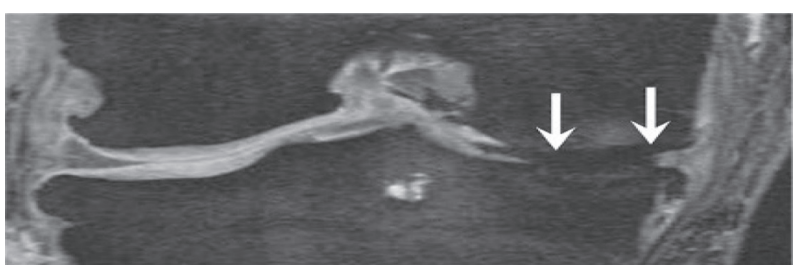

(a)

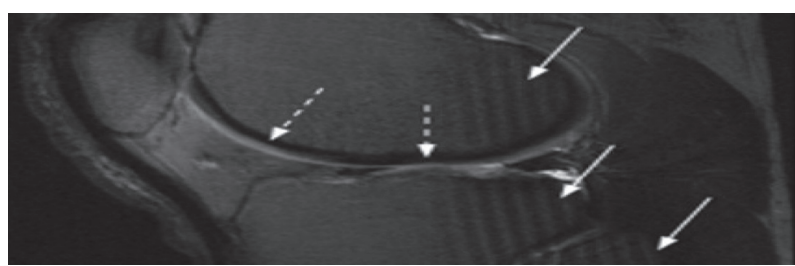

(b)

FIGURE 3: Artifacts in knee magnetic resonance image: (a) susceptibility and (b) artifacts produced by chemical (dashed arrow) and variation artifact (arrow) (reproduced from reference [11]).

physically or semiautomatically segmented, and area of interest (AOI) is estimated. There are numerous approaches for segmentation of bone and cartilage that are reviewed in this section, as illustrated in Figure 4.

\subsection{Manual and Traditional Computational Approaches.} It is often seen that the traditional and manual methods used for segmentation result in reliable outcomes. In these traditional and manual methods, the tissues are manually segmented slice by slice from magnetic resonance images. Though the precision, sensitivity, and specificity of the manual methods are considered as the gold standard and high compared to automatic and semiautomatic techniques, it requires time-consuming efforts by the experts and provides inter- or intraobserver inconsistency by different professionals [14]. These manual techniques are not practically utilized for clinical trials, for example, Figure 5 representing femur bone which is manually segmented.

The manual strategy for the identification of segmented regions from the medical image is implemented with high precision. A statistical model [15] is developed to solve the issues related to knee image segmentation. This method used 60 images for identification of knee thickness with an 


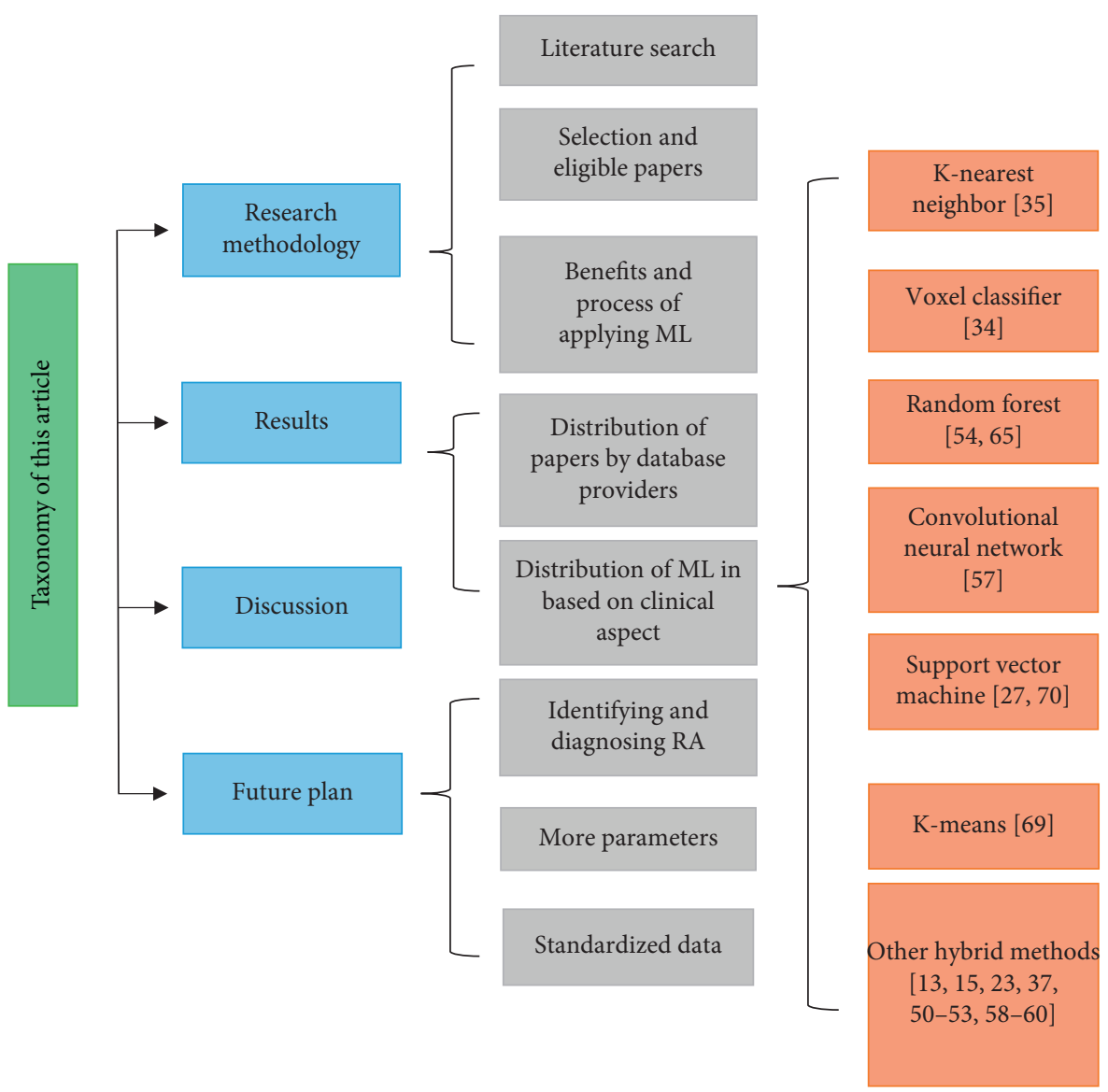

FIgURE 4: Taxonomy used in this article.

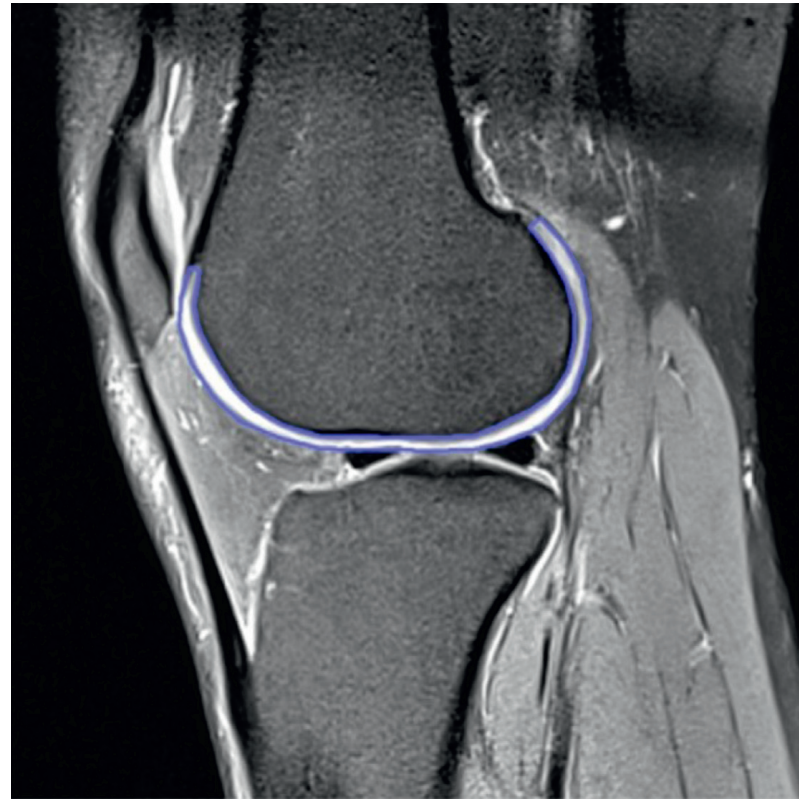

FIgURE 5: Manual segmentation of femur bone.

extreme and tiniest density of the cartilage. The method was used on the public dataset with 150 knee images [16], and mostly the data consisted of T1-weighted images with an index of $\sim 73 \%$ and $\sim 74 \%$ of femur and tibia segments, respectively.

3.1.1. Region-Based Technique. A region or area is poised of pixels of the neighbours, and the boundary is estimated by the difference between two regions. In this article, we discuss the most prevalent region-based techniques region growing and thresholding and edge-based method [17]. The initialization and process of segmentation start with some seed points. This process divides the regions based on the intensity of the pixel. The process requires only one seed point and homogenous properties of neighbouring pixels. Figure 6 represents the region growing technique, where two seed points are selected to segment the tissue.

3.1.2. Threshold or Intensity-Based Technique. The simplest and speediest method for segmentation is thresholding. The method assumes the regions with a diverse gray level. The diverse parts of the image are identified with histogram intensity. The intensity is divided into twofold: the first portion known as the foreground is having the pixel intensity higher than or equal to a threshold, second portion known as a background having the pixel intensity less than the threshold value as shown in equation: 


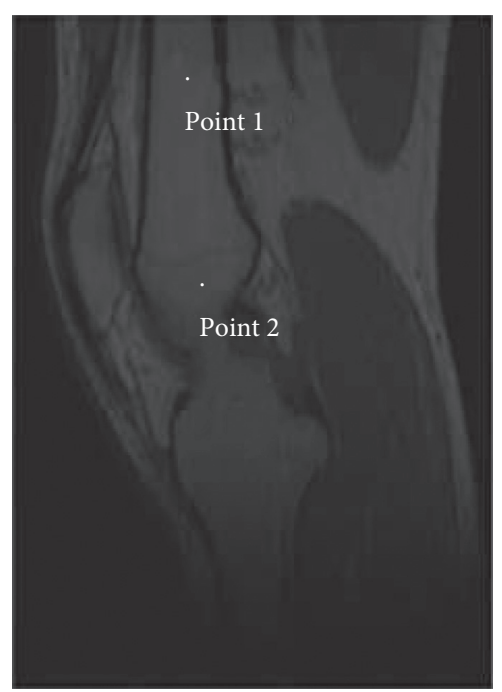

(a)

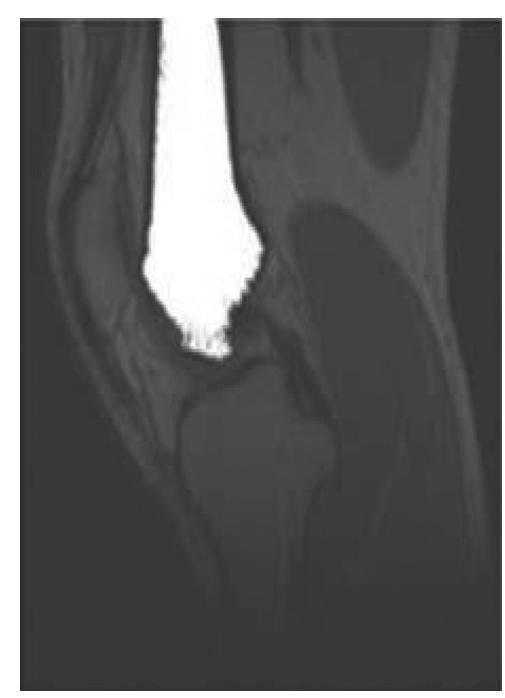

(b)

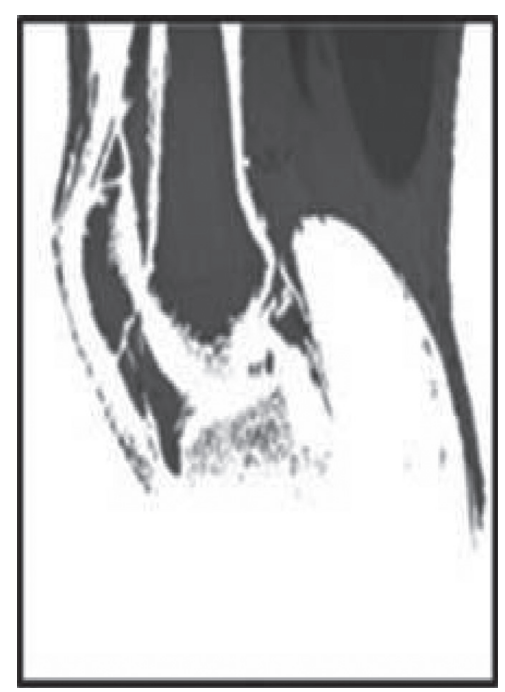

(c)

FIGURE 6: (a) Initial image with two seed points. (b) Outcome of region growing for one seed point. (c) Outcome of region growing for two seed points.

$$
f(x, y)=\left\{\begin{array}{l}
\text { foreground } g(x, y) \geq P \\
\text { background } g(x, y)<P
\end{array}\right\},
$$

where $f(x, y)$ is the pixel strength at $(x, y)$ location and $P$ is the threshold value. If the threshold value is inappropriate, then it leads to inaccurate segmentation outcomes [18]. This thresholding parameter occurs as a problem in magnetic resonance images and makes an image partition complex.

The global thresholding does not provide better results for some types of images, like the images that do not have low contrast and low pixel intensity across the image. For these types of images, the thresholding provides a better outcome in some parts of the image and fails in other parts of the image [19]. In the local thresholding, the image is partitioned into vertical and horizontal lines. The local thresholding needs more time compared to global thresholding.

3.1.3. Edge-Based Technique. The edges or boundaries are relevant information in the image. The incidence of the image is known by the number of changes in pixel strength that is utilized for identifying the different regions of those changes [20]. The automatic classification of boundaries is achieved by some high filters that avoid low-intensity data (uniform data) and preserves high-intensity data (edge data). These edges are extracted for utilization in sophisticated approaches [21]. The tissues appear to be weak or break that are not distinguishable by some methods with edgebased region and distance information.

3.1.4. Atlas or Graph-Based Technique. The gray pixels of the image are utilized to create a graph with gray indices [22]. The atlas and graphs are designed manually by experts who assign labels to the tissue structures. The segmentation process is applied for transferring the image information from labelled data to subjects. Hence, the efficiency of the image segmentation relies on image registering and resemblance between subjects and atlas. The multiatlas method $[23,24]$ that is a sequence of multiple graphs is used to collect the information in terms of color, structure, and texture of the tissues with gray values. Furthermore, the image is partitioned into several multiple small regions and segmented with foreground and background areas.

A three label approach that utilizes the atlas approach [25] for bone and cartilage segmentation is applied and evaluated on knee images. This study extensively described the performance of different classifiers, and the mean Jaccard value is $75.3 \%$ and $75.6 \%$ for femur and tibia bones. The graph method proposed in [26] offers a subject extraction with dimensionality where the weights of edges are related to boundary properties. The method evaluated the images obtained from the OAI database with a better performance parameter.

3.2. Fuzzy-Based Approaches. The fuzzy c-means approach provides a better classification of medical images. The disease is being classified into four levels based on severity which is introduced in [27]. The knee arthritis is categorized as normal, minimal, doubtful, and moderate as per the standard practice of the KL score. The morphometric measures of the knee assigned as osteoarthritis are investigated with image processing methods. The outcomes intensify and reinforce the traditional approaches with the harshness of the application. The tissues such as gray-level and white-level intensified are segmented with bias-filed corrected fuzzy C-means in [28]. A combination of the levelset and fuzzy method clusters the gray and white matter from the magnetic resonance images.

The graphical processing units are providing fast computation along with the fuzzy method that is implemented in [29]. A large dataset can be processed by accelerating the 
fuzzy c-means with GPU to tackle the computational time around 2.24 times faster. The specific region of interest (ROI) is extracted, and the active contour of the cartilage with noninterpolated areas is estimated in [30]. The identical pixels are assigned with some classes by using the membership function to detect the pathological changes in the tissue structure of the knee magnetic resonance image.

3.3. Machine Learning Approaches. In the recent past, a great effort has been employed on machine learning practices for solving the segmentation problem [31]. The unsupervised learning approach does not need training or labelling the data. The labelling of the image is accomplished by exploiting shapes in the voxel strength features. The supervised learning approach requires training for the algorithm and learns from the voxel examples. The support vector machines with multiple sets of images [32] are used for the evaluation of knee cartilage with better sensitivity. 36 different dimensional features are selected and experimented on 4 different classifiers with an AUC of $76 \%$. The hidden biomedical information is analyzed clearly for the progression of arthritis.

The subcortical segmentation method is used to construct the graph [33]. The reliable and automated segmentation of structures from magnetic resonance images is more important for shape analysis and volumetric study. The random forest classifier is used for assigning a cost for each node in the graph and evaluates the dice index and putamen measures. A method based on the K-NN strategy [34] showed successful segmentation for cartilage. The cartilage is segmented from the background that leads to false-positive values. Furthermore, the study was modified and presented with SVM [35]. The main difficulty of SVM is the distribution and independence of data instances. To incorporate the contextual data such as intensity and structural information, the ML approach builds feature vector [36] with simultaneous use of multiple images. The method utilizes the T1-weighted images for cartilage segmentation problems that are multicontrast and deposited with fat and water gradient. A method based on pattern recognition [32] for gradient data of the knee cartilage segment reports averages dice value to 0.76 .

3.4. Deep Learning Approaches. A priori knowledge of shape analysis using a convolution neural network [37] is proposed that incorporates the SSM. The method uses 40 validation and 50 subjects for the estimation of knee images. The voxel accuracy of $89 \%$ is estimated with manual adjustment, and the correlation between the developed method and the ground truth is investigated. The automated segmentation is based on the CNN approach [38]. This method applies volumetric structure on images and manually segments the magnetic resonance images. The automatic segmentation of images achieved better outcomes with a dice similarity score of 0.95 and a precision of 0.95 on femur tissue.

The contextual constrained neural network combined with level set evolution [39] is used for the segmentation of knee magnetic resonance images. The movement of the patella in the knee T2-weighted image should be normal, so tracking this with segmenting the femur and patella is required. The performance of the T1-weighted knee magnetic resonance images in terms of ASD and RMSD is estimated with the convolution network proposed in [40]. The deep learning approach has some promising issues, such as high dependency on the quality and amount of training data that tends to overfit of data [41].

The 3D deformable approach introduced in [42] uses CNN with 3D simplex deformable modeling. The method performs pixelwise, multiclass classification which has been tested on the public knee image dataset. This method provides state-of-the-art performance with superior accuracy and segmentation error of VOE. An extended version of the method is proposed in [43] for segmenting the cartilage lesion detection. The T2-weighted fast spin-echo magnetic resonance images of 175 subjects are used with CNN. The ROC and $k$ statistics were used for analyzing the performance and intraobserver detection of cartilage lesions. The volumetric assessment of knee cartilage is introduced in [44]. The dice score and VOE are estimated for different architectures using the CNN approach.

\section{Performance of Segmentation Approaches}

Most of the studies that involve segmentation of the cartilage have been evaluated by estimating the performance parameters against the ground truth. This ground truth is being prepared using manual extraction methods by an expert. To estimate the efficiency of progressive methods for estimating the cartilage boundaries, different metrics such as sensitivity, specificity, reliability, dice similarity coefficient (DSC), accuracy, and efficiency are considered from $[6,45,46]$. Based on the ground truth and developed methods, the sensitivity, specificity, DSC, and accuracy are measured using equations (3)-(6), respectively:

$$
\begin{aligned}
\text { sensitivity } & =\frac{\mathrm{TP}}{\mathrm{TP}+\mathrm{FN}}, \\
\text { specificity } & =\frac{\mathrm{TN}}{\mathrm{TN}+\mathrm{FP}}, \\
\mathrm{DSC} & =\frac{2 \mathrm{TP}}{((\mathrm{TP}+\mathrm{FP})+(\mathrm{FN}+\mathrm{TP}))}, \\
\text { accuracy } & =\frac{\mathrm{TP}+\mathrm{TN}}{\mathrm{TP}+\mathrm{TN}+\mathrm{FP}+\mathrm{FN}},
\end{aligned}
$$

where TP is true positive, i.e., area correctly labelled as cartilage area, TN is true negative, i.e., area correctly labelled as noncartilage area, FP is false positive, i.e., area incorrectly labelled as cartilage area, and FN is false negative, i.e., area incorrectly labelled as noncartilage area. These parameters are estimated with base values over the segmented areas.

Sensitivity is the parameter for a ratio of true positives and measures approximate to $100 \%$ for accurate classification of cartilage areas of ground truth and developed methods. In this parameter, the total quantity of small false negatives represents high sensitivity. The specificity 
parameter provides the ratio of true negatives that are appropriately identified through comparative analysis. A combined effect of both sensitivity and specificity reflects the best efficiency of the developed approach. Accurateness is a measure for representing the ground truth agreeing with the developed methods. It shows how many correctly classified cartilage areas are included and noncartilage areas are excluded. Other performance parameters are also considered by many researchers.

4.1. Automatic Segmentation Approaches. Automatic segmentation from magnetic resonance image is being achieved for widespread studies in rheumatoid arthritis, which makes the process that requires to be accomplished fast and consistent. Research provides more sophisticated methods to segment the images and moves towards an entirely automatic process. Once the tissues are segmented, the quantitative or measurable analysis is carried out by estimating the volume, density, and structure of the tissues. Most of the automated techniques that are grounded on voxel arrangement require a large number of training dataset. The most used machine learning approach is K-nearest neighbour applied with a multivariant classifier.

Several studies have discussed the utilization of voxel classification to segment the tissues. Although these voxel methods can segment the magnetic resonance images, the data required is the primary concern. The main limitation of this kind of method is the generation of a new dataset. The automatic methods used by different researchers with different metrics are demonstrated in Table 1. All methods discussed in the table are utilizing intensity or pixel for the computation. Generally, image segmentation can be achieved by using numerous parameters such as edges, regions, clusters, and image intensity. The threshold-based segmentation approach is limited by tissue properties such as (a) varying intensities of cartilage region, (b) low signal intensity, and (c) low visibility of tissues. The development of these techniques is still under research, and a lot yet has to be discovered. Current developments in the area of segmentation have been aimed at multiple pulse sequence of magnetic resonance images.

To automatically segment the tissues from the magnetic resonance image, the successive approach is performed with automated segmentation by extracting probabilities using the k-NN classifier. The statistical shape and models are more precise segmentation methods. However, the utilization of these methods has some limitations due to the initialization of sensitivity [62].

4.2. Semiautomatic Segmentation Approaches. This approach requires minimal user intervention during the segmentation process. These approaches are considered for reducing the efforts required for manual segmentation. These approaches with the need for human interaction complete some tasks of pattern recognition and image processing. The semiautomated approach uses some of the methods such as snake contours, gradient vectors, watershed, graph search, and region growing for segmentation. As depicted in Table 2, the methods used for semiautomatic segmentation have resulted in great success in extracting the soft and hard tissue features. A repetitive averaging filter is used for replicating Gaussian function in the deblurring process. To dehaze the images by segmentation using the super pixels technique [72], intensities are summed and the transmission map is estimated. This method preserves the features such as texture and edges of real-time images.

The semiautomated approach requires less computational time and provides an accurate outcome in the studies when compared with manual techniques, but includes the human interaction, viewer inconsistency that needs to be performed. Figure 7 represents the femur and tibia tissue segmentation using the atlas-based approach for sagittal and axial images. The method discussed in [14] provides a multicontrast image that has a rich set of the feature given for classifier using support vector machine technique. Furthermore, besides the local features, the global features are also included in the anatomical direction of femur and tibia bones. SVM is extended, and new SVM-DRF includes the independent estimation of pixels to incorporate the spatial dependency between neighbouring pixels.

\section{Discussion}

The assessment of tissues is the crucial stage in assessing the progression of rheumatoid arthritis. The segmentation process is not only needed for treatment but also used for quantitative parameter estimation. This has become a need due to complex variations related to different tissues in the knee magnetic resonance image. The segmentation techniques discussed above are broadly classified as traditional or manual, machine learning approach, deep learning approach, automatic approach, and semiautomatic approach. Manual segmentation is a tedious task and has result variability amongst professionals within the same image dataset. Semiautomatic segmentation is a less complex task compared to manual segmentation but is the same in terms of variations of manual segmentation. The intraobserver reproducibility for the density is measured in several areas of cartilage [73]. The discussed techniques are included such as region growing, active contour, and graph-cut. All these techniques require less human intervention and provide better sensitivity, specificity, and dice similarity scores. To overcome the drawback of inter- or intraobserver, many researchers came up with automated segmentation approaches that are discussed and reviewed in Section 4.

Table 3, provides a comparative analysis of other articles and this article. This paper shows an indetail insight selecting all the features related to rheumatoid arthritis. In Table 3, this review work selects synovial fluid and meniscus that is the main source for rheumatoid arthritis. The use of magnetic resonance images and machine learning approaches is the key parameter for selecting the review articles. This review gives an overview of different techniques utilized for the segmentation of tissues with resolving major challenges in the magnetic resonance image. The techniques available require more time for computation for segmentation of the 
TABLE 1: Automated segmentation approaches.

\begin{tabular}{|c|c|c|c|c|}
\hline Study & MR sample & $\begin{array}{l}\text { Number of } \\
\text { subjects }\end{array}$ & Methods used & Efficiency measures \\
\hline [47] & 3D DESS & 33 subjects & $\begin{array}{l}\text { Region growing and seed } \\
\text { selection }\end{array}$ & $\begin{array}{c}\text { DSC } 82.8 \%, 83.1 \% \text {, and } 72.6 \% \text { for femur, tibia, and patella, } \\
\text { respectively }\end{array}$ \\
\hline$[15]$ & T1-weighted 3D DESS & 20 subjects & $\begin{array}{l}\text { Side selection and coupled } \\
\text { cartilage segmentation }\end{array}$ & $\begin{array}{c}\text { DSC } 0.80 \\
\text { Sensitivity } 90.0 \\
\text { Specificity } 99.8\end{array}$ \\
\hline [48] & 3D DESS & 20 subjects & Spatial fuzzy C-mean & $\begin{array}{c}\text { DSC of femur, tibia, and patella are } 87.1,81.1 \text {, and } 84.8 \text {, } \\
\text { respectively }\end{array}$ \\
\hline [37] & DESS & 50 subjects & Statistical shape model & $\begin{array}{l}\text { Accuracy } 74 \pm 5 \\
\text { DSC } 86.1 \%\end{array}$ \\
\hline [49] & $2 \mathrm{D}$ scan & 100 subjects & Random walker algorithm & $\begin{array}{l}\text { DSC } 0.8758 \\
\text { DSC } 90.28\end{array}$ \\
\hline$[50]$ & DESS & 8 subjects & DRLSE algorithm & $\begin{array}{l}\text { Sensitivity } 91.14 \\
\text { Specificity } 99.12 \\
\text { Sensitivity } 84.2\end{array}$ \\
\hline$[35]$ & 3D DESS & 10 subjects & K-nearest neighbor & $\begin{array}{l}\text { Specificity } 99.9 \\
\text { Dice volume } 0.81\end{array}$ \\
\hline$[34]$ & 3D image & 139 subjects & Voxel classification & DSC $0.80 \pm 0.04$ \\
\hline$[51]$ & DESS & 13 subjects & $\begin{array}{l}\text { Hierarchical classifier and } \\
\text { random forest }\end{array}$ & Surface positioning errors femur $0.03 \pm 0.19$ tibia $0.10 \pm 0.17$ \\
\hline$[52]$ & $\begin{array}{l}\text { T1-weighted 3D SPGR } \\
\text { and T2-weighted GRE }\end{array}$ & 9 subjects & Three-label segmentation & Median dice coefficient femur 0.935 and tibia 0.938 \\
\hline$[53]$ & 3D SPGR & 155 subjects & $\begin{array}{l}\text { Longitudinal three label } \\
\text { segmentation }\end{array}$ & $\begin{array}{l}\text { Femur DSC } 75 \% \\
\text { Sensitivity } 78 \% \\
\text { Specificity } 99.9 \%\end{array}$ \\
\hline$[54]$ & Sagittal DESS & 88 subjects & $\begin{array}{l}\text { Convolution neural } \\
\text { network }\end{array}$ & DSC $83.8 \%$ \\
\hline$[55]$ & DESS & 40 subjects & $\begin{array}{l}\text { Active appearance model } \\
\quad \text { (statistical model) }\end{array}$ & RMSE for femur 1.49 , tibia 1.21 \\
\hline$[56]$ & PDW TSE & 14 subjects & $\begin{array}{l}\text { Distance-weighted } \\
\text { directional gradient }\end{array}$ & Intensity level, $2 \sim 4$ \\
\hline$[57]$ & T1-weighted & 30 subjects & $\begin{array}{l}\text { Multiatlas segmentation } \\
\text { constrained graph }\end{array}$ & Femur average surface distance $0.36 \mathrm{~mm}$ \\
\hline [58] & 2D ASM & 140 subjects & Rigid multiatlas registration & $\begin{array}{l}\text { Dice volume tibia } 0.8 \text { and femur } 0.87 \\
\text { DSC }\end{array}$ \\
\hline$[59]$ & T1-weighted SPGR & 20 subjects & Statistical method & $\begin{array}{l}\text { of femur } 0.947 \text {, tibia } 0.949 \text {, and patella } 0.909 \text {. Specificity of } \\
\text { femur } 0.988 \text {, tibia } 0.993 \text {, and patella } 0.996 \text {. }\end{array}$ \\
\hline$[60]$ & T1-weighted FS & 14 subjects & $\begin{array}{l}\text { Validation of statistical } \\
\text { method }\end{array}$ & Meniscus DSC 0.75 , sensitivity 0.72 , and specificity 1.00 \\
\hline$[61]$ & T1-weighted & 100 subjects & $\begin{array}{l}\text { Coarse to fine method with } \\
\text { min-cut }\end{array}$ & DSC of femur 0.947, and tibia 0.968 \\
\hline
\end{tabular}

TABle 2: Semiautomated segmentation approaches.

\begin{tabular}{lcccc}
\hline Study & MR sample & $\begin{array}{c}\text { Number of } \\
\text { subjects }\end{array}$ & Methods used & Efficiency measures \\
\hline$[63]$ & PDW SPAIR & 12 subjects & Random forest & Femur DSC $94.9 \%$ \\
Tibia DSC $92.5 \%$ \\
Accuracy $72 \%$ \\
{$[27]$} & T1-weighted axial & 103 subjects & Support vector machine & Dice volume 0.82 \\
{$[58]$} & CCBR & 159 subjects & PLDS method & Error $-6.53 \%$ \\
{$[64]$} & 3D SGPR & 4 subjects & Region growing & Mean error -0.57 \\
{$[65]$} & T1-weighted & 5 subjects & Active shape model & Interobserver 3.3 to 13.6 \\
{$[66]$} & Flash 3D & 15 subjects & B-spline with manual & adjustment \\
{$[45]$} & 3DMR & 20 subjects & Active contour & Thickness 0.996 and 0.998 for femur and tibia \\
{$[4]$} & SPGR & 7 subjects & Watershed transformation $89.5 \%$ \\
& & & Sensitivity $90 \%$ \\
\end{tabular}


TABLE 2: Continued.

\begin{tabular}{|c|c|c|c|c|}
\hline Study & MR sample & $\begin{array}{c}\text { Number of } \\
\text { subjects }\end{array}$ & Methods used & Efficiency measures \\
\hline$\overline{[23]}$ & 3D DESS & 320 slices & Graph-cut algorithm & DSC $94.3 \%$ \\
\hline [67] & Flash GRE & 50 subjects & $\begin{array}{l}\text { K-means with manual } \\
\text { adjustment }\end{array}$ & $\begin{array}{c}\text { DSC } 0.77 \text { and } 0.80 \\
\text { Sensitivity } 83.1 \text { and } 85.3 \\
\text { Specificity } 99.9 \text { and } 099.9 \text { for femur and tibia bones, } \\
\text { respectively }\end{array}$ \\
\hline [68] & DESS & 17 subjects & Support vector machine & DSC patella 0.82 , tibia 0.83 , and femur 0.86 \\
\hline [23] & 3T MR images & 10 subjects & Graph-cut method & DSC 0.943 \\
\hline [13] & 3D DESS & 12 subjects & Active contour model & Root mean square $0.8 \%$ to $1.3 \%$ \\
\hline [69] & 3D DESS & 10 subjects & Mesh morphing approach & Mean S.D of femur 0.87 , tibia 0.40 and, patella 0.53 \\
\hline [70] & T1-weighted & 15 subjects & Watershed method & Cartilage volume tibia $3.3 \mathrm{~mm}$ \\
\hline
\end{tabular}
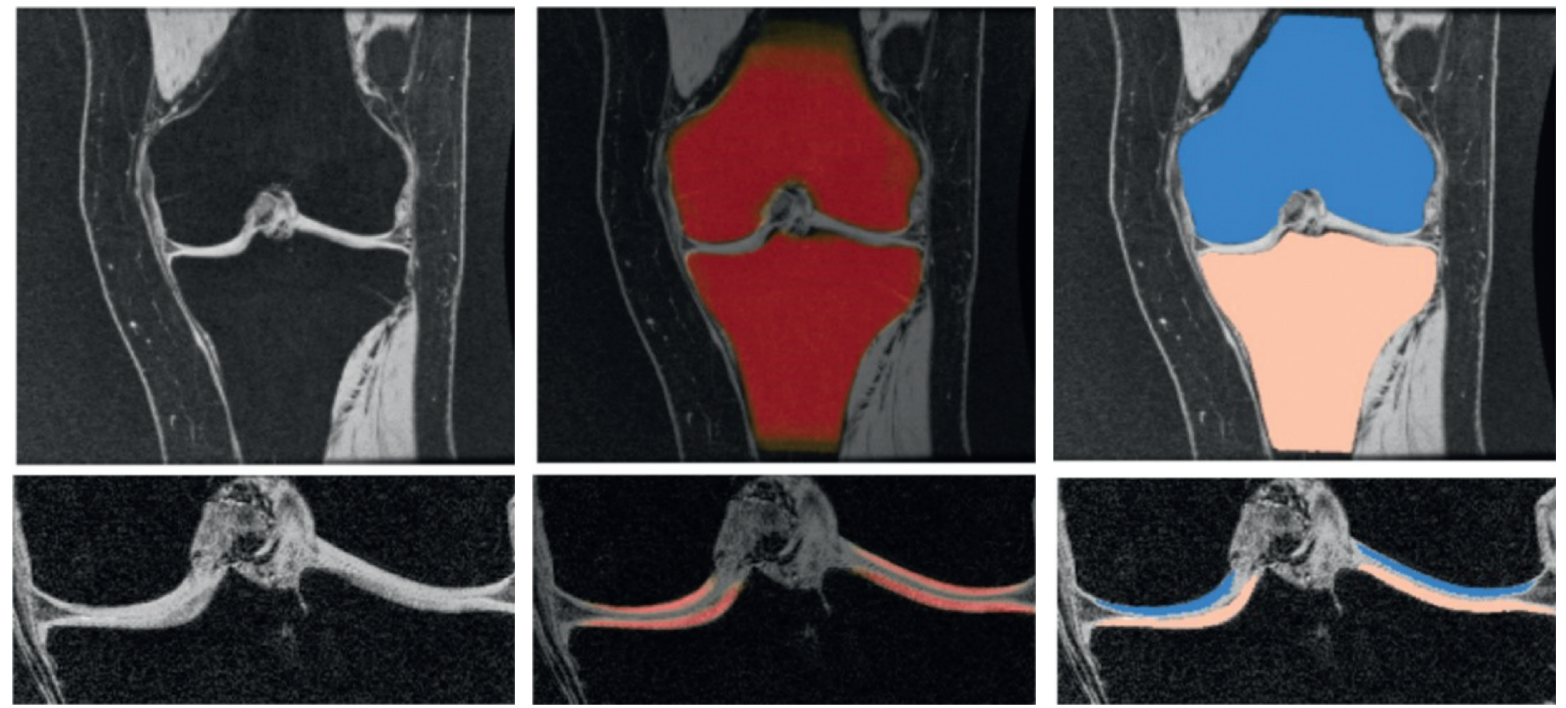

FiguRE 7: First row: segmentation of knee bone (left: original image, middle: automated segmentation, and right: segmented bone). Second row (left: original image, middle: automated segmentation, and right: cartilage segmented) (reproduced from reference [25]).

TABLE 3: Comparative analysis with other published review articles.

\begin{tabular}{|c|c|c|c|c|c|c|c|}
\hline \multirow{2}{*}{ Article } & \multirow{2}{*}{$\begin{array}{c}\text { Datatype considered } \\
\text { Magnetic resonance } \\
\text { imaging }\end{array}$} & \multirow{2}{*}{$\begin{array}{l}\text { Medical disease } \\
\text { considered } \\
\text { Machine learning }\end{array}$} & \multicolumn{5}{|c|}{ Classification and summarization of existing work } \\
\hline & & & Cartilage & Meniscus & $\begin{array}{c}\text { Ligaments and } \\
\text { lesions }\end{array}$ & $\begin{array}{l}\text { Patella, tibia, and } \\
\text { femur bone }\end{array}$ & $\begin{array}{l}\text { Synovial } \\
\text { fluid }\end{array}$ \\
\hline$[74]$ & $\sqrt{ }$ & $\sqrt{ }$ & $\mathrm{X}$ & $\mathrm{X}$ & $\sqrt{ }$ & $\mathrm{X}$ & $\mathrm{X}$ \\
\hline$[75]$ & $\sqrt{ }$ & $\sqrt{ }$ & $\sqrt{ }$ & $\mathrm{X}$ & $\mathrm{X}$ & $\mathrm{X}$ & $\mathrm{X}$ \\
\hline$[76]$ & $\sqrt{ }$ & $\sqrt{ }$ & $\mathrm{X}$ & $\sqrt{ }$ & $\mathrm{X}$ & $\mathrm{X}$ & $\mathrm{X}$ \\
\hline$[77]$ & $\mathrm{X}$ & $\sqrt{ }$ & $\mathrm{X}$ & $\mathrm{X}$ & $\mathrm{X}$ & $\mathrm{X}$ & $\sqrt{ }$ \\
\hline$[78]$ & $\sqrt{ }$ & $\sqrt{ }$ & $\mathrm{X}$ & $\mathrm{X}$ & $\mathrm{X}$ & $\sqrt{ }$ & $\mathrm{X}$ \\
\hline $\begin{array}{l}\text { This } \\
\text { article }\end{array}$ & $\sqrt{ }$ & $\sqrt{ }$ & $\sqrt{ }$ & $\sqrt{ }$ & $\sqrt{ }$ & $\sqrt{ }$ & $\sqrt{ }$ \\
\hline
\end{tabular}

entire knee magnetic resonance image. Some of the automated and semiautomated approaches discussed above are highlighted with the main strengths and drawbacks. However, the growing rate of data and increasing rate of computational power make the machine learning and deep learning approaches most promising for future applications concerning IoT applications [79-83].

\section{Conclusion}

This systematic review approximates the magnetic resonance imaging key parameters reminiscent of rheumatoid arthritis. Our nature of the study selects sensitivity, specificity, and dice similarity index for an approximation of imaging results. However, from the results of our study, more frequent 
parameters for rheumatoid arthritis disease such as synovial fluid volume, meniscus volume, the dissimilar structure of tibia, femur, and tears of ligaments. Different segmentation techniques are considered for review with pros and cons of themselves that may increase the productivity of a novel hybrid approach.

Our future work includes selecting more parameters that are directly related to rheumatoid arthritis. The synovial fluid and cystic lesions are the less considered features than the cartilage and other bone tissues. These considered features may be used in the future for targeted treatment, in those most required for rheumatoid arthritis diagnosis.

\section{Conflicts of Interest}

The authors declare that they have no conflicts of interest.

\section{Acknowledgments}

This work was supported in part by Zayed University, Office of Research under Grant no. R18088.

\section{References}

[1] S. Castañeda, J. A. Roman-Blas, R. Largo, and G. HerreroBeaumont, "Subchondral bone as a key target for osteoarthritis treatment," Biochemical Pharmacology, vol. 83, no. 3, pp. 315-323, 2012.

[2] D. Lubar, P. H. White, L. F. Callahan et al., "A national public health agenda for osteoarthritis 2010," Seminars in Arthritis and Rheumatism, vol. 39, no. 5, pp. 323-326, 2010.

[3] A. O. Akinpelu, T. O. Alonge, B. A. Adekanla, and A. C. Odole, "Prevalence and pattern of symptomatic knee osteoarthritis in Nigeria: a community-based study," The Internet Journal of Allied Health Sciences and Practice, vol. 7, no. 3, pp. 1-7, 2009.

[4] V. Grau, A. U. J. Mewes, M. Alcañiz, R. Kikinis, and S. K. Warfield, "Improved watershed transform for medical image segmentation using prior information," IEEE Transactions on Medical Imaging, vol. 23, no. 4, pp. 447-458, 2004.

[5] B. Zhang, "Computer-aided knee joint magnetic resonance image segmentation-a survey," 2018, https://arxiv.org/abs/ 1802.04894 .

[6] D. Kumar, A. Gandhamal, S. Talbar, and A. F. M. Hani, "Knee articular cartilage segmentation from MR images: a review," ACM Computing Surveys, vol. 51, no. 5, p. 1, 2019.

[7] B. S. Bentley and R. V. Hill, "Assessing macroscopic and microscopic indicators of osteoarthritis in the distal interphalangeal joints: a cadaveric study," Clinical Anatomy, vol. 20, no. 7, pp. 799-807, 2007.

[8] T. G. Williams, A. P. Holmes, M. Bowes et al., "Measurement and visualisation of focal cartilage thickness change by MRI in a study of knee osteoarthritis using a novel image analysis tool," The British Journal of Radiology, vol. 83, no. 995, pp. 940-948, 2010.

[9] P. R. Kornaat, S. B. Reeder, S. Koo et al., "MR imaging of articular cartilage at $1.5 \mathrm{~T}$ and $3.0 \mathrm{~T}$ : comparison of SPGR and SSFP sequences," Osteoarthritis and Cartilage, vol. 13, no. 4, pp. 338-344, 2005.

[10] R. Stahl, R. Krug, D. A. C. Kelley et al., "Assessment of cartilage-dedicated sequences at ultra-high-field MRI: comparison of imaging performance and diagnostic confidence between 3.0 and 7.0 T with respect to osteoarthritis-induced changes at the knee joint," Skeletal Radiology, vol. 38, no. 8, pp. 771-783, 2009.

[11] P. Lang, F. Noorbakhsh, and H. Yoshioka, "MR imaging of articular cartilage: current state and recent developments," Radiologic Clinics of North America, vol. 43, no. 4, pp. 629639, 2005.

[12] A. Aprovitola and L. Gallo, "Knee bone segmentation from MRI: a classification and literature review," Biocybernetics and Biomedical Engineering, vol. 36, no. 2, pp. 437-449, 2016.

[13] M. H. Brem, P. K. Lang, G. Neumann et al., "Magnetic resonance image segmentation using semi-automated software for quantification of knee articular cartilage-initial evaluation of a technique for paired scans," Skeletal Radiology, vol. 38, no. 5, pp. 505-511, 2009.

[14] K. Zhang, W. Lu, and P. Marziliano, "Automatic knee cartilage segmentation from multi-contrast MR images using support vector machine classification with spatial dependencies," Magnetic Resonance Imaging, vol. 31, no. 10, pp. 1731-1743, 2013.

[15] H. Seim, D. Kainmueller, H. Lamecker, M. Bindernagel, J. Malinowski, and S. Zachow, "Model-based auto-segmentation of knee bones and cartilage in MRI data," in Proceedings of the 13th International Conference on Medical Image Computing and Computer Assisted Intervention, Beijing, China, 2010.

[16] T. Heimann and B. Morrison, "Segmentation of knee images: a grand challenge," in Proceedings of the 2010 MICCAI Workshop on Medical Image Analysis for the Clinic, Beijing, China, 2010.

[17] A. Norouzi, M. S. M. Rahim, A. Altameem et al., "Medical image segmentation methods, algorithms, and applications," IETE Technical Review, vol. 31, no. 3, pp. 199-213, 2014.

[18] P. K. Sahoo, S. Soltani, and A. K. C. Wong, "A survey of thresholding techniques," Computer Vision, Graphics, and Image Processing, vol. 41, no. 2, pp. 233-260, 1988.

[19] A. Rad, M. S. Mohd Rahim, A. Rehman, A. Altameem, and T. Saba, "Evaluation of current dental radiographs segmentation approaches in computer-aided applications," IETE Technical Review, vol. 30, no. 3, pp. 210-222, 2013.

[20] Y. Yin, X. Zhang, R. Williams, X. Wu, D. D. Anderson, and M. Sonka, "LOGISMOS-layered optimal graph image segmentation of multiple objects and surfaces: cartilage segmentation in the knee joint," IEEE Transactions on Medical Imaging, vol. 29, no. 12, pp. 2023-37, 2010.

[21] M. S. Swanson, J. W. Prescott, T. M. Best et al., "Semi-automated segmentation to assess the lateral meniscus in normal and osteoarthritic knees," Osteoarthritis and Cartilage, vol. 18, no. 3, pp. 344-353, 2010.

[22] J.-G. Lee, S. Gumus, C. H. Moon, C. K. Kwoh, and K. T. Bae, "Fully automated segmentation of cartilage from the MR images of knee using a multi-atlas and local structural analysis method," Medical Physics, vol. 41, no. 9, Article ID 092303, 2014.

[23] H. Shim, S. Chang, C. Tao, J.-H. Wang, C. K. Kwoh, and K. T. Bae, "Knee cartilage: efficient and reproducible segmentation on high-spatial-resolution MR images with the semiautomated graph-cut algorithm method," Radiology, vol. 251, no. 2, pp. 548-556, 2009.

[24] L. Shan, C. Charles, and M. Niethammer, "Automatic multiatlas-based cartilage segmentation from knee MR images," in Proceedings of the 2012 9th IEEE International Symposium on Biomedical Imaging (ISBI), Barcelona, Spain, 2012. 
[25] L. Shan, C. Zach, C. Charles, and M. Niethammer, "Automatic atlas-based three-label cartilage segmentation from MR knee images," Medical Image Analysis, vol. 18, no. 7, pp. 1233-1246, 2014.

[26] Y. Boykov, O. Veksler, and R. Zabih, "Fast approximate energy minimization via graph cuts," IEEE Transactions on Pattern Analysis and Machine Intelligence, vol. 23, no. 11, pp. 1222-1239, 2001.

[27] A. Alkan, "Analysis of knee osteoarthritis by using fuzzy c-means clustering and SVM classification," Scientific Research and Essays, vol. 6, no. 20, pp. 4213-4219, 2011.

[28] P. Agarwal, S. Kumar, R. Singh, P. Agarwal, and M. Bhattacharya, "A combination of bias-field corrected fuzzy $\mathrm{C}$-means and level set approach for brain MRI image segmentation," in Proceedings of the 2015 2nd International Conference on Soft Computing and Machine Intelligence (ISCMI), Hong Kong, China, 2016.

[29] M. Al-Ayyoub, A. M. Abu-Dalo, Y. Jararweh, M. Jarrah, and M. A. Sa'd, "A GPU-based implementations of the fuzzy C-means algorithms for medical image segmentation," The Journal of Supercomputing, vol. 71, no. 8, pp. 3149-3162, 2015.

[30] J. Kubicek and M. Penhaker, "Fuzzy algorithm for segmentation of images in extraction of objects from MRI," in Proceedings of the 2014 International Conference on Advances in Computing, Communications and Informatics (ICACCI), Delhi, India, 2014.

[31] S. Koo, B. Hargreaves, T. Andriacchi, and G. Gold, "Automatic segmentation of articular cartilage from MRI: a multi-contrast and multi-dimensional approach," in Proceedings of the 16th Scientific Meeting of the International Society for Magnetic Resonance in Medicine, Toronto, Canada, 2008.

[32] Y. Du, R. Almajalid, J. Shan, and M. Zhang, "A novel method to predict knee osteoarthritis progression on MRI using machine learning methods," IEEE Transactions on NanoBioscience, vol. 17, no. 3, pp. 228-236, 2018.

[33] Z. Guo, S. Kashyap, M. Sonka, and I. Oguz, "Machine learning in a graph framework for subcortical segmentation," Medical Imaging 2017: Image Processing, vol. 10133, 2017.

[34] J. Folkesson, E. B. Dam, O. F. Olsen, P. C. Pettersen, and C. Christiansen, "Segmenting articular cartilage automatically using a voxel classification approach," IEEE Transactions on Medical Imaging, vol. 26, no. 1, pp. 106-115, 2007.

[35] J. Folkesson, O. F. Olsen, P. Pettersen, E. Dam, and C. Christiansen, "Combining binary classifiers for automatic cartilage segmentation in knee MRI," Lecture Notes in Computer Science, Springer, vol. 3765, pp. 230-239, Berlin, Germany, 2005.

[36] K. Zhang, J. Deng, and W. Lu, "Segmenting human knee cartilage automatically from multi-contrast MR images using support vector machines and discriminative random fields," in Proceedings of the 2011 18th IEEE International Conference on Image Processing, Brussels, Belgium, 2011.

[37] F. Ambellan, A. Tack, M. Ehlke, and S. Zachow, "Automated segmentation of knee bone and cartilage combining statistical shape knowledge and convolutional neural networks: data from the osteoarthritis initiative," Medical Image Analysis, vol. 52, pp. 109-118, 2019.

[38] C. M. Deniz, S. Xiang, R. S. Hallyburton et al., "Segmentation of the proximal femur from MR images using deep convolutional neural networks," Scientific Reports, vol. 8, no. 1, pp. 1-14, 2018.
[39] H.-C. Lan, T.-R. Chang, W.-C. Liao, Y.-N. Chung, and P.-C. Chung, "Knee MR image segmentation combining contextual constrained neural network and level set evolution," in Proceedings of the 2009 IEEE Symposium on Computational Intelligence in Bioinformatics and Computational Biology, Nashville, TN, USA, 2009.

[40] H. Lee, H. Hong, and J. Kim, "BCD-NET: a novel method for cartilage segmentation of knee MRI via deep segmentation networks with bone-cartilage-complex modeling," in Proceedings of the 2018 IEEE 15th International Symposium on Biomedical Imaging (ISBI 2018), Washington, DC, USA, April 2018.

[41] J.-G. Lee, S. Jun, Y.-W. Cho et al., "Deep learning in medical imaging: general overview," Korean Journal of Radiology, vol. 18, no. 4, pp. 570-584, 2017.

[42] F. Liu, Z. Zhou, H. Jang, A. Samsonov, G. Zhao, and R. Kijowski, "Deep convolutional neural network and 3D deformable approach for tissue segmentation in musculoskeletal magnetic resonance imaging," Magnetic Resonance in Medicine, vol. 79, no. 4, pp. 2379-2391, 2018.

[43] F. Liu, Z. Zhou, A. Samsonov et al., "Deep learning approach for evaluating knee MR images: achieving high diagnostic performance for cartilage lesion detection," Radiology, vol. 289, no. 1, pp. 160-169, 2018.

[44] A. Raj, S. Vishwanathan, B. Ajani, K. Krishnan, and H. Agarwal, "Automatic knee cartilage segmentation using fully volumetric convolutional neural networks for evaluation of osteoarthritis," in Proceedings of the 2018 IEEE 15th International Symposium on Biomedical Imaging (ISBI 2018), Washington, DC, USA, 2018.

[45] C. Kauffmann, P. Gravel, B. Godbout et al., "Computer-aided method for quantification of cartilage thickness and volume changes using MRI: validation study using a synthetic model," IEEE Transactions on Biomedical Engineering, vol. 50, no. 8, pp. 978-988, 2003.

[46] J. Tang, S. Millington, S. T. Acton, J. Crandall, and S. Hurwitz, "Surface extraction and thickness measurement of the articular cartilage from MR images using directional gradient vector flow snakes," IEEE Transactions on Bio-Medical Engineering, vol. 53, no. 5, pp. 896-907, 2006.

[47] C. N. Öztürk and S. Albayrak, "Automatic segmentation of cartilage in high-field magnetic resonance images of the knee joint with an improved voxel-classification-driven regiongrowing algorithm using vicinity-correlated subsampling," Computers in Biology and Medicine, vol. 72, pp. 90-107, 2016.

[48] C. Ahn, T. D. Bui, Y.-w. Lee, J. Shin, and H. Park, "Fully automated, level set-based segmentation for knee MRIs using an adaptive force function and template: data from the osteoarthritis initiative," BioMedical Engineering Online, vol. 15, no. 1, pp. 1-14, 2016.

[49] P. R. Desai and I. Hacihaliloglu, "Enhancement and automated segmentation of ultrasound knee cartilage for early diagnosis of knee osteoarthritis," in Proceedings of the 2018 IEEE 15th International Symposium on Biomedical Imaging (ISBI 2018), Washington, DC, USA, April 2018.

[50] A. Gandhamal, S. Talbar, S. Gajre, R. Razak, A. F. M. Hani, and D. Kumar, "Fully automated subchondral bone segmentation from knee MR images: data from the osteoarthritis initiative," Computers in Biology and Medicine, vol. 88, pp. 110-125, 2017.

[51] S. Kashyap, H. Zhang, K. Rao, and M. Sonka, "Learning-based cost functions for 3-D and 4-D multi-surface multi-object segmentation of knee MRI: data from the osteoarthritis 
initiative," IEEE Transactions on Medical Imaging, vol. 37, no. 5, pp. 1103-1113, 2018.

[52] L. Shan, C. Zach, and M. Niethammer, "Automatic three-label bone segmentation from knee MR images," in Proceedings of the 2010 IEEE International Symposium on Biomedical Imaging: From Nano to Macro, Rotterdam, Netherlands, 2010.

[53] L. Shan, C. Charles, and M. Niethammer, "Longitudinal threelabel segmentation of knee cartilage," in Proceedings of the 2013 IEEE 10th International Symposium on Biomedical Imaging, San Francisco, CA, USA, 2013.

[54] A. Tack, A. Mukhopadhyay, and S. Zachow, "Knee menisci segmentation using convolutional neural networks: data from the osteoarthritis initiative," Osteoarthritis and Cartilage, vol. 26, no. 5, pp. 680-688, 2018.

[55] G. Vincent, C. Wolstenholme, I. Scott, and M. Bowes, "Fully automatic segmentation of the knee joint using active appearance models," Medical Image Analysis for the Clinic: A Grand Challenge, CreateSpace, Scotts Valley, CA, USA, 2010.

[56] F. Huang, X. Chen, D. Ye, and S. Hertel, “A distance weighted directional gradient method for fully automatic bone segmentation of knee MRI," in Proceedings of the 16th Scientific Meeting of the International Society of Magnetic Resonance in Medicine, Toronto, Canada, 2008.

[57] C. Chu, J. Bai, X. Wu, and G. Zheng, "MASCG: multi-atlas segmentation constrained graph method for accurate segmentation of hip CT images," Medical Image Analysis, vol. 26, no. 1, pp. 173-184, 2015.

[58] E. B. Dam, "Simple methods for scanner drift normalization validated for automatic segmentation of knee magnetic resonance imaging - with data from the osteoarthritis initiative," 2017, https://arxiv.org/abs/1712.08425.

[59] J. Fripp, S. Ourselin, S. K. Warfield, and S. Crozier, "Automatic segmentation of the bones from MR images of the knee," in Proceedings of the 2007 4th IEEE International Symposium on Biomedical Imaging: From Nano to Macro, pp. 336-339, Arlington, VA, USA, 2007.

[60] J. Fripp, P. Bourgeat, C. Engstrom, S. Ourselin, S. Crozier, and O. Salvado, "Automated segmentation of the menisci from MR images," in Proceedings of the 2009 IEEE International Symposium on Biomedical Imaging: From Nano to Macro, Boston, MA, USA, 2009.

[61] S. Hyun, S. Lee, I. Dong, and S. Uk, "Automatic bone segmentation in knee MR images using a coarse-to-fine strategy," vol. 8314, pp. 1-6, 2012.

[62] E. B. Dam, J. Folkesson, P. C. Pettersen, and C. Christiansen, "Semi-automatic knee cartilage segmentation," in Proceedings of the Medical Imaging 2006: Image Processing, San Diego, CA, USA, 2006.

[63] M. Marčan and I. Voiculescu, "Unsupervised segmentation of MRI knees using image partition forests," in Proceedings of the Medical Imaging 2016: Biomedical Applications in Molecular, Structural, and Functional Imaging, vol. 9788, San Diego, CA, USA, 2016.

[64] A. Piplani, D. G. Disler, T. J. Holmes, T. R. Mccauley, and J. P. Cousins, "Articular cartilage volume in the knee: semiautomated determination from three-dimensional reformations of MR images," Radiology, vol. 198, no. 3, pp. 855-859, 1996.

[65] S. Solloway, C. E. Hutchinson, J. C. Waterton, and C. J. Taylor, "The use of active shape models for making thickness measurements of articular cartilage from MR images," Magnetic Resonance in Medicine, vol. 37, no. 6, pp. 943-952, 1997.
[66] D. T. Sandwell, "Thermal isostasy: response of a moving lithosphere to a distributed heat source," Journal of Geophysical Research: Solid Earth, vol. 87, no. B2, pp. 1001-1014, 1982.

[67] P. Dodin, J. Pelletier, J. Martel-Pelletier, and F. Abram, "Automatic human knee cartilage segmentation from 3-D magnetic resonance images," IEEE Transactions on Biomedical Engineering, vol. 57, no. 11, pp. 2699-2711, 2010.

[68] J. Fripp, S. Crozier, S. K. Warfield, S. Ourselin, and S. Ourselin, "Automatic segmentation and quantitative analysis of the articular cartilages from magnetic resonance images of the knee," IEEE Transactions on Medical Imaging, vol. 29, no. 1, pp. 55-64, 2010.

[69] M. A. Baldwin, J. E. Langenderfer, P. J. Rullkoetter, and P. J. Laz, "Development of subject-specific and statistical shape models of the knee using an efficient segmentation and mesh-morphing approach," Computer Methods and Programs in Biomedicine, vol. 97, no. 3, pp. 232-240, 2010.

[70] F. K. Patel and M. Singh, "Segmentation of cartilage from knee MRI images using the watershed algorithm," International Journal of Advance Research, Ideas and Innovations in Technology, vol. 4, no. 2, pp. 1727-1730, 2018.

[71] H. Hassan, A. K. Bashir, R. Abbasi, W. Ahmad, and B. Luo, "Single image defocus estimation by modified Gaussian function," Transactions on Emerging Telecommunications Technologies, vol. 30, no. 6, pp. 1-14, 2019.

[72] H. Hassan, A. K. Bashir, M. Ahmad et al., "Real-time image dehazing by superpixels segmentation and guidance filter," Journal of Real-Time Image Processing, pp. 1-21, 2020.

[73] A. Saygllı and S. Albayrak, "A new computer-based approach for fully automated segmentation of knee meniscus from magnetic resonance images," Biocybernetics and Biomedical Engineering, vol. 37, no. 3, pp. 432-442, 2017.

[74] N. Bien, P. Rajpurkar, R. L. Ball et al., "Deep-learning-assisted diagnosis for knee magnetic resonance imaging: development and retrospective validation of MRNet," PLoS Medicine, vol. 15, no. 11, Article ID e1002699, 2018.

[75] B. G. Ashinsky, M. Bouhrara, C. E. Coletta et al., "Predicting early symptomatic osteoarthritis in the human knee using machine learning classification of magnetic resonance images from the osteoarthritis initiative," Journal of Orthopaedic Research, vol. 35, no. 10, pp. 2243-2250, 2017.

[76] A. Saygllı and S. Albayrak, "An efficient and fast computeraided method for fully automated diagnosis of meniscal tears from magnetic resonance images," Artificial Intelligence in Medicine, vol. 97, no. 13, pp. 118-130, 2019.

[77] R. Hemalatha, V. Vijaybaskar, and T. Thamizhvani, "Automatic localization of anatomical regions in medical ultrasound images of rheumatoid arthritis using deep learning," Proceedings of the Institution of Mechanical Engineers, Part H: Journal of Engineering in Medicine, vol. 233, no. 6, pp. 657667, 2019.

[78] Z. Zhou, G. Zhao, R. Kijowski, and F. Liu, "Deep convolutional neural network for segmentation of knee joint anatomy," Magnetic Resonance in Medicine, vol. 80, no. 6, pp. 2759-2770, 2018.

[79] N. Tsafack, S. Sankar, B. Abd-El-Atty et al., "A new chaotic map with dynamic analysis and encryption application in internet of health things," IEEE Access, vol. 8, Article ID 137731, 2020.

[80] M. Al-Maitah, A. A. AlZubi, and A. Alarifi, "An optimal storage utilization technique for IoT devices using sequential machine learning," Computer Networks, vol. 152, pp. 98-105, 2019. 
[81] A. A. AlZubi, A. Alarifi, and W. Alnumay, "Machine learningbased channel analysis for user concentric optical switching networks," Circuits, Systems, and Signal Processing, vol. 39, no. 2, pp. 1178-1194, 2020.

[82] S. Deep, X. Zheng, A. Jolfaei, D. Yu, P. Ostovari, and A. Kashif Bashir, "A survey of security and privacy issues in the internet of things from the layered context," Transactions on Emerging Telecommunications Technologies, Article ID e3935, 2020.

[83] Z. Zheng, T. Wang, J. Wen, S. Mumtaz, A. K. Bashir, and S. H. Chauhdary, "Differentially private high-dimensional data publication in internet of things," IEEE Internet of Things Journal, vol. 7, no. 4, pp. 2640-2650, 2020. 